\title{
Web Profile Recommendations for Safe Surfing and Ethical Usage
}

\author{
V. Dhilip Kumar ${ }^{1}$, Gaurav Kumar ${ }^{1}$, Ajay Singh Patel ${ }^{1}$, Neeraj Chaurasiya ${ }^{1}$, Kemal Polat ${ }^{2, *}$ \\ ${ }^{1}$ Department of CSE, Vel Tech Rangarajan Dr.Sagunthala $R$ \& D Institute of Science and Technology, Chennai \\ ${ }^{2}$ Department of Electrical and Electronics Engineering, Bolu Abant Izzet Baysal University, Bolu 14280, Turkey \\ *Corresponding Author: kpolat@ibu.edu.tr
}

How to cite this paper: V. Dhilip Kumar, Gaurav Kumar, Ajay Singh Patel, Neeraj Chaurasiya, Kemal Polat (2021). Web Profile Recommendations for Safe Surfing and Ethical Usage. Journal of the Institute of Electronics and Computer, 3, 53-60. https://doi.org/10.33969/JIEC.2021.31004.

Received: July 26, 2021

Accepted: August 13, 2021

Published: August 16, 2021

Copyright (C 2021 by author(s) and Institute of Electronics and Computer. This work is licensed under the Creative Commons Attribution International License (CC BY 4.0).

http://creativecommons.org/licenses/by/4.0/

(c) (i) Open Access

\begin{abstract}
People are using the internet for all their day-to-day activities like shopping, banking, mailing, etc. The term censored is a kind of spoofing website which is used to steal important information. Therefore, there is a need for an efficient prevention mechanism to find out the censored web page among the legitimate web pages. This paper proposes a study on identifying censored websites by developing an effective algorithm with domain features of URL. Detecting proxies by URL is done by implementing domain pattern matching. We found that the detector accuracy and speed, reflecting in response efficacy as perceived by users, censored websites as a great source of phishing and malicious activities that users have no knowledge about. Data from government-issued is used to detect censored sites. Security of browsers is the concern with users having low-level security settings.
\end{abstract}

\section{Keywords}

Censored, detecting proxies, spoofing, security

\section{Introduction}

This paper aims to make a Chromium Extension that will help end-users with Web Surfing Monitoring; it is a Browser plugin written using web languages like HTML, CSS, and JavaScript. This Extension provides monitoring and recommendation to the user and includes info like Security Monitoring and recommendation for safety, Website Monitoring, Censored and Unsafe website warnings. Extensions are mostly zipped files of HTML, CSS, JavaScript, and other web surfing files that customize the browsing experience. Pop-up is built using web technology and can use the same API as the website provides to the browser. Extensions have an extensive range of functional possibilities. They can easily modify web content and UI of the website as 
users want and interact with or extend and change the behavior of the website itself. Consider extensions of the gateway to make the browser experience the most enhanced and easy user. We use Google Chrome as a preferred browser as it is commonly used in India and several different countries. Google Chrome extension is a small software that provides additional functionality to the browser; Extensions are commonly known as a plugin. The need for a plugin is to enhance the functionality of the web browser.

Chromium is a preferred browser, and alternation is necessary for other browsers like Firefox, UC Browser, Opera Mini, Microsoft Browser v. X, etc. after alternation, there may be some slight difference in the UI, and maybe some functionality difference will be there.

\section{Impact of Internet}

Now-a-days internet making more effective societies across the planet. But parallel simultaneously, the power and effectiveness of the internet are misused by the people, groups, organizations, and corporations. And the people are worried about the privacy of their logs, which is making an unprecedented impact on society. Society is impacted by various ways such as educational resources, social media platforms, web-based apps, online shopping platforms, e-businesses, net-marketing, research, and automation of social skills positively and negatively. There is a chunk of people in society can't expect a happier day without accessing the internet in their daily life. These days internet influenced everything in the core of society from student to teacher, Entrepreneurs to the client, seller to buyer, and human to robots. No one spare from internet technology. Even when a baby cries his/her mother plays song or video in mobile/iPad, kids stop crying and smiling face. This is huge. You can it is either positive or negative.

How the internet was impacting on mental health by bullying. Bullying is just a part of growing up, and it can have a serious impact on someone's health and wellbeing. To overcome this negative impact, we proposed this extension idea, which can warn us at the time of excess use and also protect our children from opening censored and unwanted web pages, and this will also help users to save from the cross checker and phishing websites by stealing the users' data and mass level of online fraud. Internet becomes the problem of health issues where the majority of society is impacted by it. Politics is also influenced by the internet too; in the last election in the USA, Cambridge Analytica was blamed for influencing voters in favor of a specific political party. Indian users are switching towards the internet with a huge number and risk factors and impacts on society. Below this graph shows how internet users in our country are increasing. This is creating impact on society, but this is very 
helpful and can't be ignored, and this is to be the opportunity, as we know that this era is the Cyberworld, and anyone can't afford to keep away from it. These should be the opportunities and make ensure safety, security, and privacy to the users. This was also helping to make boost to the economy and making ease to live such as human development, social development and connectivity with the world within the matter of click, and this major area can't be ignored, instead of ignorance government should make ensure safety and security of people by the certain development of policy and technology. Some psychological reports suggested that "Internet addiction" is Gambling addiction. By this fact, we can understand that how big this issue is, and how much this impacting the society and influencing the people life as well.

\section{Impact of mental health on bullying}

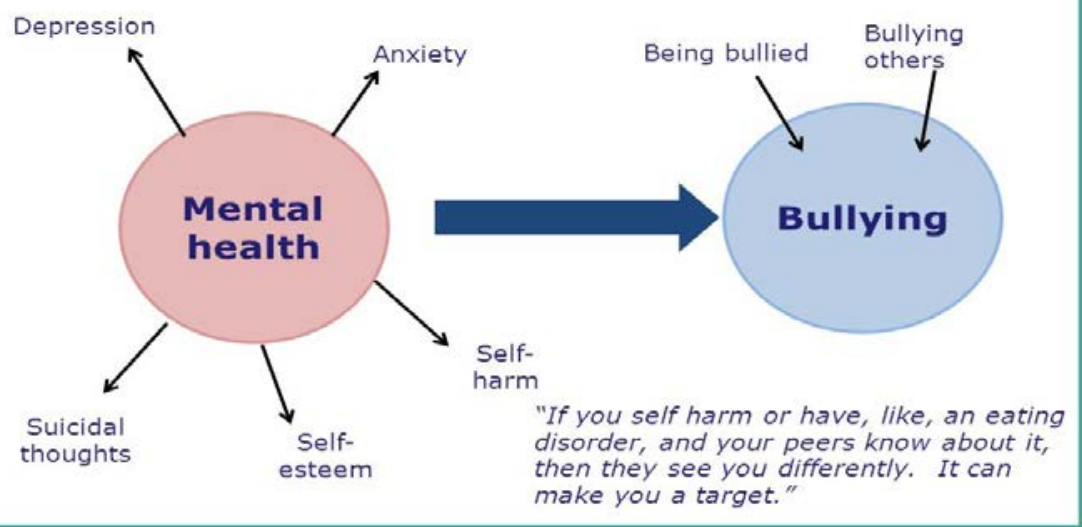

Figure 1. Impact of mental health on bullying

\section{Application of This System}

\section{A. Website Monitoring}

This Extension can provide information about the site; the Extension can retrieve the security information and trust level of the website and be able to recommend a website like it.

\section{B. Time based categorization}

This Extension can compare similar websites and is able to provide the best results among them. This Plug-in is also Analysis the Time used for each website and provides results accordingly. In addition, it categorizes history for better view and understanding.

\section{Basic Security Management and recommendation}


This Extension can give proper security recommendations as the danger from the website is a common thing. Most of the time, the end-user doesn't know what to do in those types of situations as if the Extension provides the security measures and recommendations. Then, it will be very easy for the user to surf the internet without any difficulties. However, some warnings will be provided as a notification and changing the extension icon's color.

\section{Censored and Unsafe Website Warning}

This Plug-in has a small local cache built-in itself in the browser. It is filled with all the Censored and unsafe Website domains that Wikipedia openly provides, and this Extension is filled with all the updated list of the unsafe website. So whenever the user visits such a website the Extension will pop up warning and give proper suggestions for it to close.

\section{E. History Monitoring}

This Extension will be capable for analysis of History and active Time and date for the website so it can be easily retrieved by the end-user without collecting their personal data and. We need access to the history, which is easily provided by History API. History API is the API provided by the browsers themselves to access by the Extension. The history is easily available and visible in the extension bar down the menu bar right side, but the user doesn't care what type of website he is surfing. We can provide that information in an easily analyzed way.

\section{How This System Works}

This Extension can run on Chromium browser and use chromium API, which provides apps with a unique feature and extends their functionality. This system can do Time Profile Analysis to maintain the profile of the end-user and be able to give recommendations. The Basic Security Management and Recommendation use Privacy API to prevent from harmful websites and can give warning via changing icon color and capable of preventing to visit those harmful websites, we are also using History API to keep track of visited website by the end-user and can analysis those and will show proper security measures for it. Various APIs are used in this system are: Chromium API, History API, Privacy API, Top sites API, Notification API, Page Action API, Browser Action API. The Extension is built using the JavaScript asynchronous method, which can do multiple tasks simultaneously and are interdependent; due to this, the Extension can reduce the processing time and make the user a manageable workload and easy scheduling.

In this architecture explanation about how the whole system works, when user start browsing or searching then enters URL then browser engine is already Extension it will do some process like domain name capture, active time of the user and malicious 
V. Dhilip Kumar et al.

servers and unwanted or harmful websites. Then the analysis is to be done by the local cache storage of the browser. Then, it will start output performance on the user interface, such as action display, profile analysis, and recommendation.

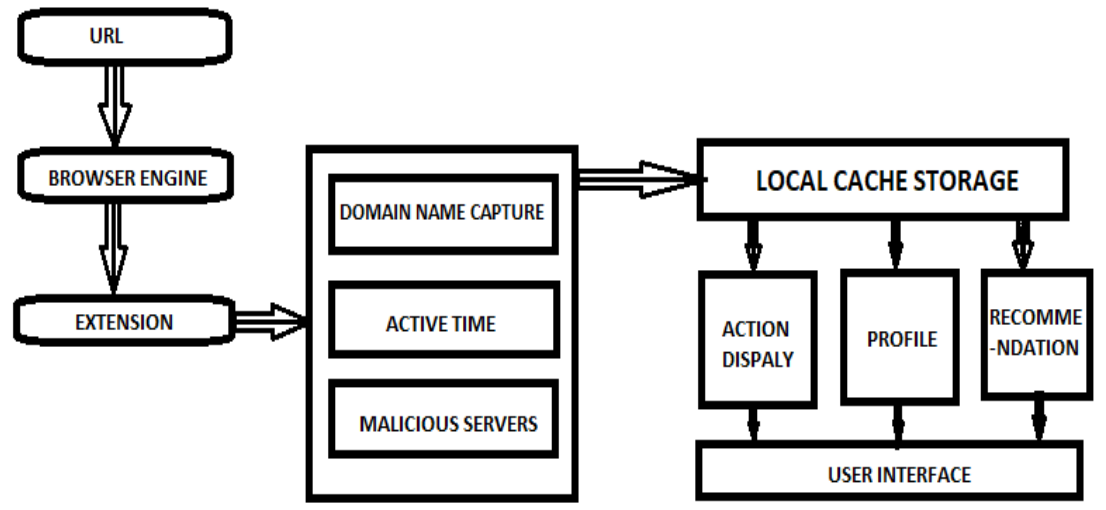

Figure 2. The flowchart of the proposed model

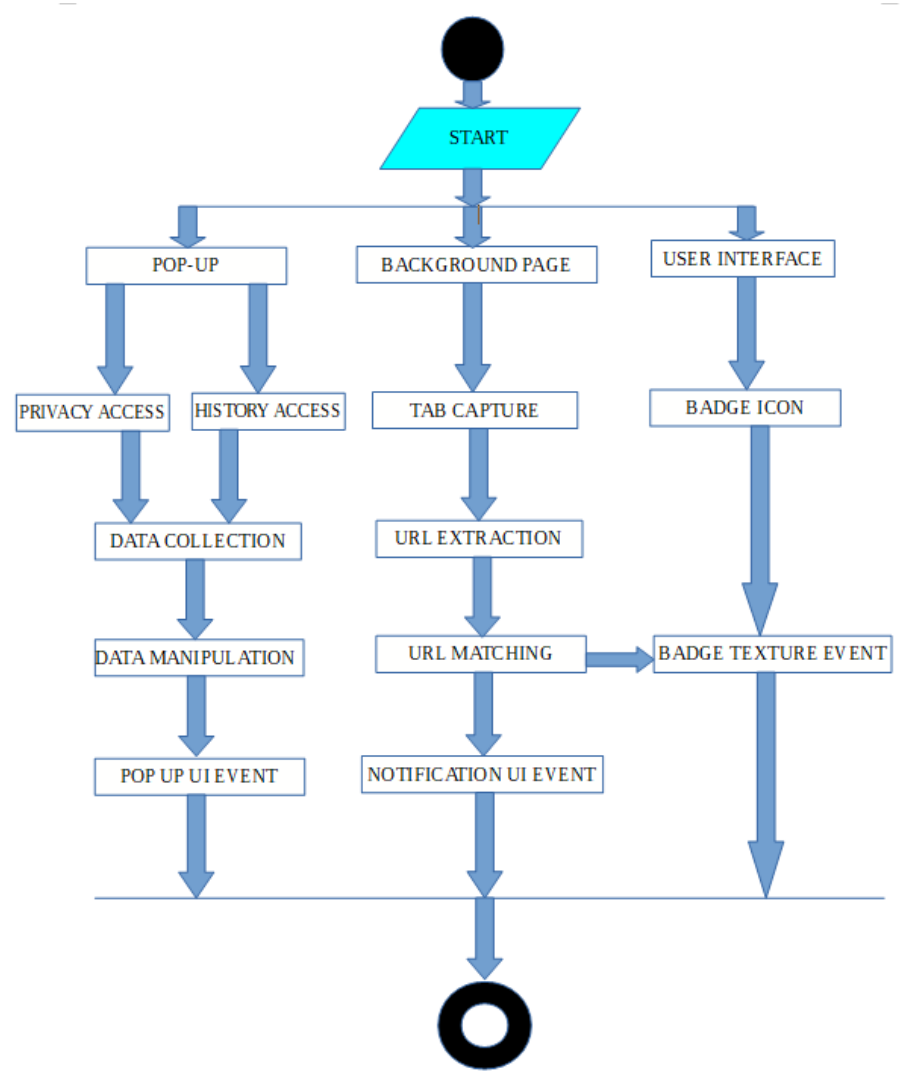

Figure 3. The working scheme of the proposed system 


\section{The advantages of the proposed system are given as follows:}

1) To keep track of the surfing habit of the end-user, so the user can manage their time on surfing habits.

2) The Extension can show notifications about unsafe and censored websites, so can the user will get a warning before visiting it.

3) The Extension can manage the date and time profile for the user so the end-user can access the history provided in an accessible manner.

4) The Extension can capture and show the recently and most-used websites by the user and provide on the top in a list from which the user can access and open it quickly.

5) The history and date-time profile is given in two separate lists and can easily be toggled by clicking on it, and the separate profiles are Today and This week.

6) The date for visiting the website is managed in four separate lists as a link, typed, reload, and blocked.

7) Security recommendation is given to the end-user through the main UI of the Extension, so the user can easily manage their security.

The Phishing and Unsafe websites update must give manually every several months, and the accuracy will be depending on the update of the Phishing websites. As the number of phishing websites is increasing day by day and covering all of it is generally a big problem in this and a new phishing website and is not known yet, it can be dangerous for the user. This Extension is failed to cover some security features like a virus from the mail, firewall control, switching off the Extension from the extension setting of the browser, and downloading the virus intensely by the enduser; in this all above-stated situation, the Extension will fail to provide its full features as it can. This Extension is developed by taking Google Chrome as a preferred browser and alternation is necessary for other browsers like Firefox, UC Browser, Opera Mini, Microsoft Browser v. X, etc. after alternation, there may be some slight difference in the UI and maybe some functionality difference will be there.

\section{Conclusion}

The proposed Extension is able to run successfully in all the updated chromium browser and with all the latest updated system, the Extension is developed using HTML, CSS, and JavaScript it is fully compatible with any operating system using chromium browser, the Extension is readily available on Chrome Store and can be easily downloaded and installed in the system, the system is able to do the functionality for the user and the functionality are Websites Monitoring, In the website monitoring the user is able to see the primarily used websites by the user and 
V. Dhilip Kumar et al.

able to see the recent visited tabs, so easy for the user to access the recently used tabs, another functionality is time categorized analysis in this as the user able to analysis the history with perfect timing and date, and also it will provide he information of history up to a week or two, this Extension is also available with embedded Basic Security Management and Recommendation which will provide the information about the basic security like, alternate error page enabled, Auto fill Credit Card, Password Saving Enabled, Safe browsing enabled, Search Suggest Enabled, Third Party Cookies Allowed, Do not Track Enabled, Network Prediction Enabled, and WebRTC IP Handling Policy, all this security measures are given to the end user so he can easily monitor that what type of security he is getting. The proposed Extension is using the API (Application Program Interface) to exclusively increase the functionality of the Extension, APIs like Chromium API, History API, Privacy API, TopsitesAPI, Notification API, PageAction API, BrowserAction API.

\section{Conflicts of Interest}

The authors declare that they have no conflict of interest.

\section{References}

[1] www.klientsolutech.com/how-internet-impact-on-society-positively-andnegatively/

[2] Allen, N.J. and Meyer, J.P. The measurement and antecedents of affective, continuance and normative commitment to the organization. J. Occupational Psych. 63, (1990), 1-18.

[3] Open Web Application Security Project, XSS (Cross- Site Scripting), Prevention Cheat Sheet, 2011; https:// www.owasp.org/index.php/XSS_(Cross_Site_Scripting)_ Prevention_Cheat_Sheet.

[4] S. Fogie et al., XSS Attacks: Cross Site Scripting Exploits and Defense, Syngress, 2007. 3. N. Li et al., "Perturbation-Based User-Input-Validation Testing of Web Applications," J. Systems and Software, Nov. 2010, pp. 2263-2274.

[5] H. Shahriar and M. Zulkernine, "MUTEC: Mutation-Based Testing of Cross Site Scripting," Proc. 5th Int'l Workshop Software Eng. for Secure Systems (SESS 09), IEEE, 2009, pp. 47-53.

[6] M.S. Lam et al., "Securing Web Applications with Static and Dynamic Information Flow Tracking," Proc. 2008 ACM SIGPLAN Symp. Partial Evaluation and Semantics-Based Program Manipulation (PEPM 08), ACM, 2008, pp. 3-12.

[7] G. Wassermann and Z. Su, "Static Detection of Cross-Site Scripting Vulnerabilities," Proc. 30th Int'l Conf. Software Eng. (ICSE 08), ACM, 2008, pp. 171-180. 
[8] Heritrix, Internet Archive's open-source, extensible, web-scale, archival-quality web crawler project, http://crawler.archive.org/

[9] A SURVEY ON SQL INJECTION: VULNERABILITIES, ATTACKS, AND PREVENTION TECHNIQUES Diallo Abdoulaye Kindy and Al-SakibKhan Pathan Department of Computer Science, International Islamic University Malaysia, Malaysia. 